\title{
Continuous Quality Improvement Applied to Outpatient Health Care Delivery in Displaced Persons Camps
}

\author{
Paul B. Spiegel, Ellen Lynch \& Narendra M. Patel
}

\begin{abstract}
Continuous Quality Improvement (CQI) has been shown to be a highly effective approach for the evaluation and management of hospitals in developed countries, but it has barely begun to be utilised in less developed countries. This article defines the principles and the main tools of CQI and then applies them to the postemergency phase of a displaed persons camp situation, specifically towards improving the utilisation and the quality of care in an outpatient department.
\end{abstract}

\section{Résumé}

L'Accroissement Continu de la Qualité s'est révélé une approche très efficace pour l'évaluation et la gestion des hôpitaux dans les pays développés, mais n'aà toutes fins pratiques jamaisétémise à profit dans les pays moins développés. Cet article définit les principes et les principaux instruments, Accroissement Continu de la Qualité, et les appliqueà la phase post-urgente dans la situation des camps pour personnes déplacées. L'attention porteplusspécifiquementsur

Dr. Paul Spiegel was at the time of writing a Research Associate at Johns Hopkins School of Hygiene and Public Healthand Senior Associate of the Center for Refugee and Disaster Studies.

Ellen Lynch is a graduate of the Johns Hopkins University School of Nursing (BSN (RN)) and School of Hygiene and Public Health (MPH). In September 1999, she joined the US Agency for International Development as a Foreign Service Officer in the Global Bureau's Center for Population, Health and Nutrition.

Narendra Patel is a graduate of the Johns Hopkins University School of Hygiene and Public Health (MPH). In August of 1998, he joined the University of Illinois College of Medicine to pursue clinical training and is currently in his second year of studies. les possibilités d'amélioration de l'utilisation des services et de la qualité des soins en clinique externe.

\section{Introduction}

Continuous Quality Improvement (CQI) is an effective method of management and evaluation and its usage is becomingmore predominant within the field of health care. In the 1940's, Dr. W. Edwards Deming introduced the concept that improving quality by objective methods would result in competitive advantage(Deming 1986). Today one can observe its usage within hospitals, clinics, health maintenance organizations and laboratories, yet CQI has yet to be applied to the delivery of health care in a displaced persons (DP) camp setting(DP refers to refugees and internally displaced persons). This article applies the principles and tools of $\mathrm{CQI}$ in order to assess the utilisation and quality of care of outpatient departments(OPD) in a composite DP camp (this camp represents a combination of various camps in which the authors have managed and visited over a number of years throughout Africa and Asia). Over the past two decades, through numerous United Nations' (UN) and non-governmental organizations' (NGOs) experiences in DP camp settings, many commonalities have been observed, and from these, certain guidelines and recommendations have been established (Sandler and Jones 1987, Perrin 1996, Desenclos 1993, CDC 1992, Steering Committee 1998, Médecins 1997). For instance, many DP camps around the world are set up in a similar manner and have similar health care priorities. The UN has established an essential drug list (IDA 1990) in order to appropriately and effectively respond to the illnesses that commonly manifest in DP camps due to overcrowded situations, poor nutritional status, and inadequate public services (i.e. measles, diarrhoea, respiratory tract infections, and malaria in endemic areas). These similarities allow the framework outlined in this article to be applicable in most DP settings, with specific adaptations according to the local culture, the situation on the ground, and the data generated by employing CQI tools.

In this article, the tools of CQI are applied to the process of managing an OPD in a DP camp. The first stage consists of a comprehensive evaluation of the OPD in an inclusive manner as possible, comprising all levels of its health care workers, criticalcustomers (i.e. everyone involved in the process from all stages of caregivers to the patients and the indigenous community), as well as the representatives of the host government. The next stage involves the gathering and evaluation of data from various aspects of the existing processes in the OPD. The information collected will allow the CQI team to fashion a specific, concrete plan to improve and/or re-engineer processes in order to achieve the desired improvements and objectives. Periodic and continuous evaluation will be necessary to ensure continuous improvement of processes and outcomes as well as to account for changes in the local situation. This analysis concentrates upon the post-emergency phase of a DPcamp, where the situation is more stable than in the emergency phase, and hencemore suitable for the application of CQI.

\section{Background}

\section{Continuous Quality Improvement}

Quality of care should be defined according to the health care giver's level of 
skill and the patient's and community's level of expectation. Until recently, CQI, as applied in the health care setting, was primarily used in hospitals in developed countries according to standards set by accrediting agencies. In the 1980 's, the process was extended to include primary health care in Western countries (Bender 1993). Soon afterwards, WHObegan applying the process of quality assurance by developing international protocols for treatment of certain diseases (i.e. respiratory tract infections and diarrhea), and then expanded the concept to systematic analysis of health care systems in developing countries. CQI is an evolutionary process and may be defined as a structured and systematic approach for creating organisation-wide participation in planning and implementing continuous improvements in quality (Whetsell 1991). The four tenets of quality assurance, as stated by the Quality Assurance Project, which began in 1990 in order to develop and implement sustainable approaches for improving the quality of health care in less developed countries, are the following (Brown 1992):

1. CQI is oriented toward meeting the needs and expectations of the patient and community.

2. CQI focuses on systems and processes.

3. CQI uses data to analyse service delivery processes.

4. CQI encourages a team approach to problem solving and quality improvement.

Effective and simple tools are used for process analysis. The main analytic tools include flowcharts, cause and effect (fishbone) diagrams, brainstorming, decision matrices and story boarding. The major statistical tools include histograms, pareto charts, run charts, check sheets, scatter diagrams, and control charts (Brassard 1994).

Improving the quality of health care need not increase the cost of the existing system, and often becomes a cost-saving measure. In fact, CQI may often be the only alternative for over burdened health care systems, not only in developing countries, but all over the world.
One of the CQIobjectives is to maximise efficiency and effectiveness from existing systems and resources (Bender 1993).

\section{Displaced Persons Camps}

There are usually four power structures in DP camps: the United Nations High Commission for Refugees (UNHCR), the DPs themselves (usually represented by a DP committee), the NGO's, and the host government (IFRC 1996). Depending upon the decision being made, all or some of these groups should be contacted and the issues discussed before programs are implemented or changed. Currently, this inclusion principle is rarely followed. The time for implementing CQI in a DP camp is during the post-emergency phase, when the situation is more stable and the fundamental health care infrastructure has already been organized.

\section{Functioning of an OPD in a DP Camp}

Health care workers (HCWs) who treat patients in the OPD have variable levels of training which are often rudimentary. In nearly all cases, the NGOs provide on-the-job training to these workers. They are taught case-definitions and case treatment protocols, developed by the NGOs, the UN agencies or the host government for specific diseases, and are expected to follow them (Desenclos 1993). All HCWs should have a disease register which generally records the name, age, sex, location of person in camp, symptoms/signs, diagnosis and treatment prescribed for each patient seen. A patient usually has a patient card on which the HCW records the symptoms/signs, diagnosis and treatment. He is normally required to retain this card in order to be seen at the OPD in the future. When a patient arrives at the OPD, he is triaged, and then waits to see $\mathrm{HCW}$. When an emergency case is identified, the patient is immediately seen by a HCW. Depending upon the number of patients, the wait in the OPD can be minutes to many hours, and sometimes the non-urgent cases may not receive care that day at all. On aver- age, a HCW in an average OPD in a DP camp would care for anywhere between 50 to 200 patients per day. In a DP camp of 30,000 people in the post-emergency phase, there are usually between 5-10 HCWs working in an OPD. In general, diagnoses are made primarily upon history and a cursory physical exam, and then a treatment protocol is followed.

\section{Methods}

A team of three persons, comprised of an expert in CQI, a physician with extensive experience in different phases of many refugee and IDP camps, and a registered nurse with years of experience in international development and emergencies, analysed the processes of numerous different OPDs in DP camps throughout Asia and Africa.

Acomposite OPD in a DP camp, combining the most common processes of the OPDs, was developed using CQI tools. CQI principles and tools were then applied to the existing processes of this OPD (specifically, flowcharts, brainstorming, nominal group technique, and a cause and effect (fishbone) diagram). In order to find common problems and create potential solutions. The authors attempted to be as inclusive as possible and consider the points of view from the health care workers, critical customers (patients), NGOs, UN and the host country (local persons as well as the government). After analysis of the OPD, concensus occurred on certain key processes which were then examined and proposals suggested as to how to improve the efficiency and quality of the care delivered in the OPD.

\section{Results}

\section{Determination of the Objectives of the Analysis}

The Nominal Group Technique was employed in order to create the objectives of the analysis. This technique attempts to provide everyone in the team an equal voice in the problem selection following specific steps which will not be outlined here. The following objectives were agreed upon: 
1. To explain and apply CQI principles and tools to an OPD in a DP camp.

2. To increase the efficiency of the OPDby :

a. decreasing overusage of repeat health care seekers by implementation of new processes to control access.

b. modifying the DP's expectations of the type of health care offered.

c. modifying the treatments and referral patterns of the health care workers working in the OPD.
3. To improve the population's reported satisfaction of the health care services provided, given the limited resources of the UN and NGOs.

4. Toprevent long-term dependency of the population on the health care provided by the OPD, in order not to deter the ultimate goal of repatriation of the DPs.

\section{Chart 1: Flowchart of Original OPD Processes in DP Camp}

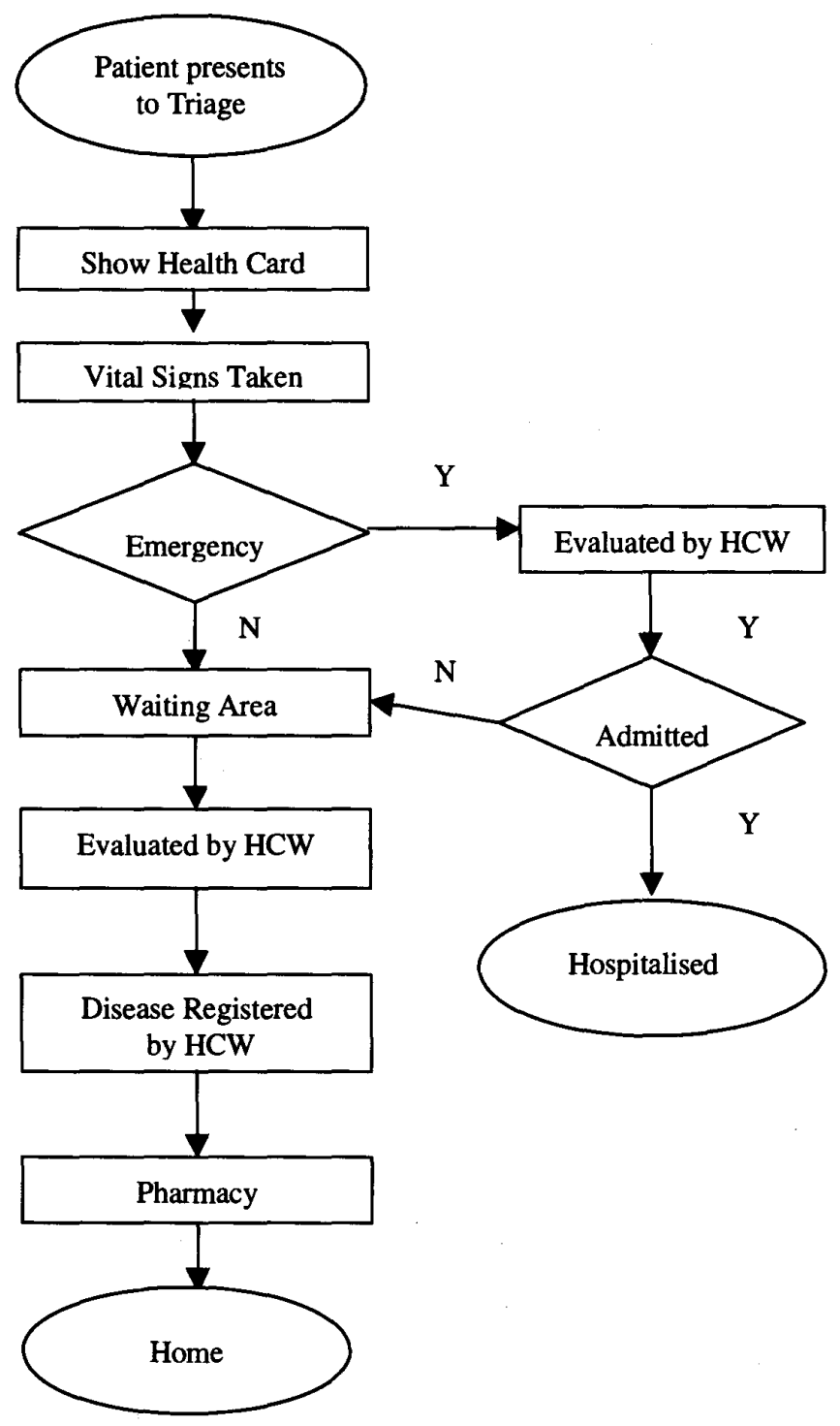

\section{Recording the Existing Processes of the OPD}

The existing processes of the OPD are analysed and portrayed in the flowchart below (Chart 1 ) in order to have a clearer understanding of the existing OPD processes and to allow for its dissection and analysis. The usage of a flowchart allows for the identification of an actual or ideal path that a product or service follows in order to identify any deviations.

\section{Determination of the Causes of Over-Utilisation of the OPD}

The cause and effect (fishbone) diagram (Chart 2) allows detailed analysis of the causes of over-utilisation of the OPD. This breakdown and subsequent clear portrayal of the relationships between the causes and effects allows for proper analysis and planning in order to correct an existing process or re-engineer a new process. A fishbone diagram represents the relationship between some effect and all the possible causes influencing it. It illustrates the various causes affecting a process by sorting out and relating them.

\section{Data Collection and Analysis}

After an unstructured brainstorming session, a concrete plan was developed and agreed upon by all members of the team regarding the type of data needed, collection methods for the data and analytic techniques in order to identify opportunities to improve the efficiency and quality of the OPD processes in the DP camps. A flowchart is shown for easy visualization of these methods (Chart 3). Brainstorming allows inclusion of all dimensions of a problem or solution without boundaries. It is used to help a group create as many ideas as possible in a short time period. 


\section{Chart 2: Cause and Effect Diagram:}

\section{Determination of the Causes of Over-Utilisation of the OPD}

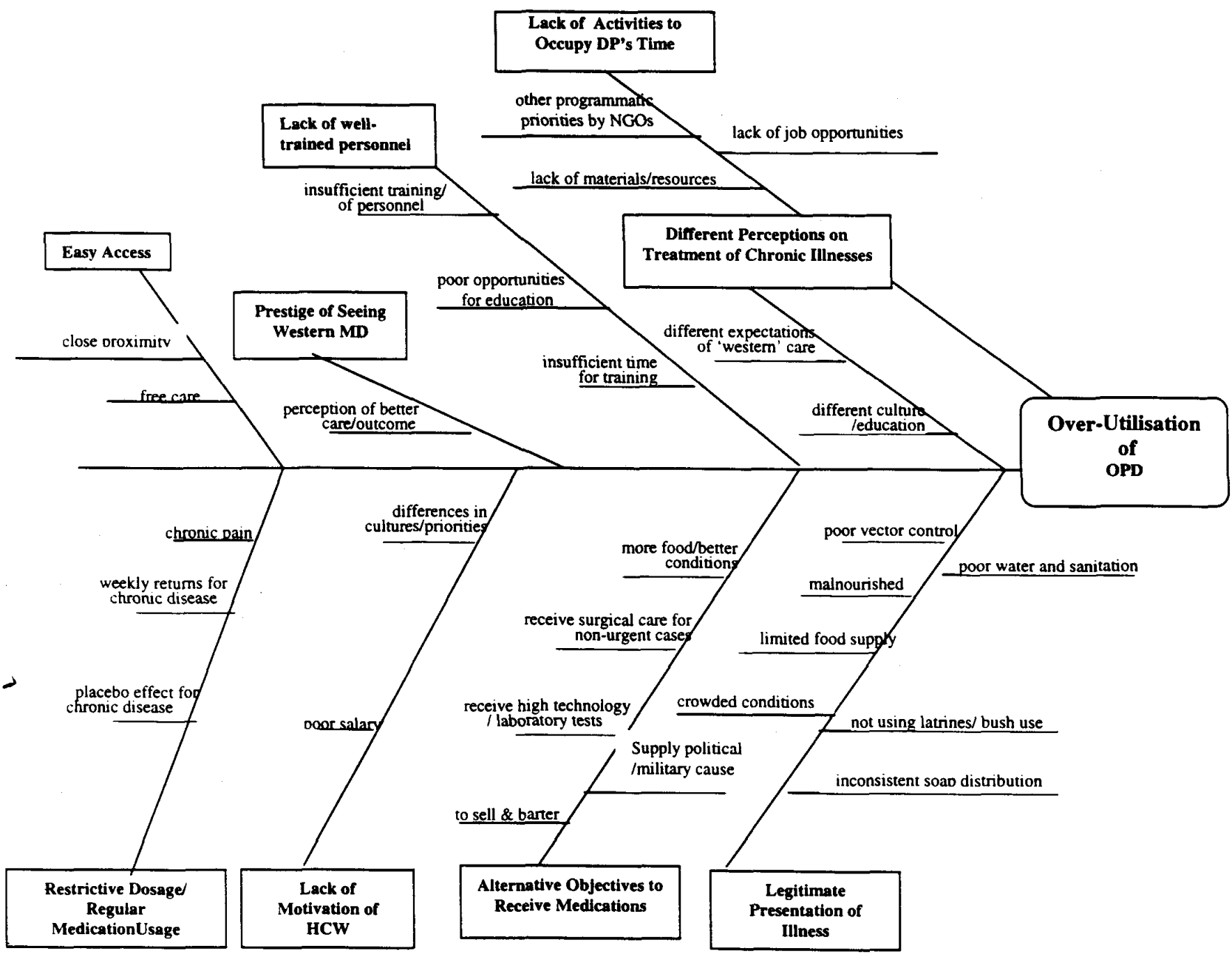

(C) Paul B. Spiegel, Ellen Lynch and Narendra M. Patel, 2000. This open-access work is licensed under a Creative Commons AttributionNonCommercial 4.0 International License, which permits use, reproduction and distribution in any medium for non-commercial purposes, provided the original author(s) are credited and the original publication in Refuge: Canada's Journal on Refugees is cited. 


\section{Chart 3 : Flow Chart of Improved OPD Processes in DP Camp}

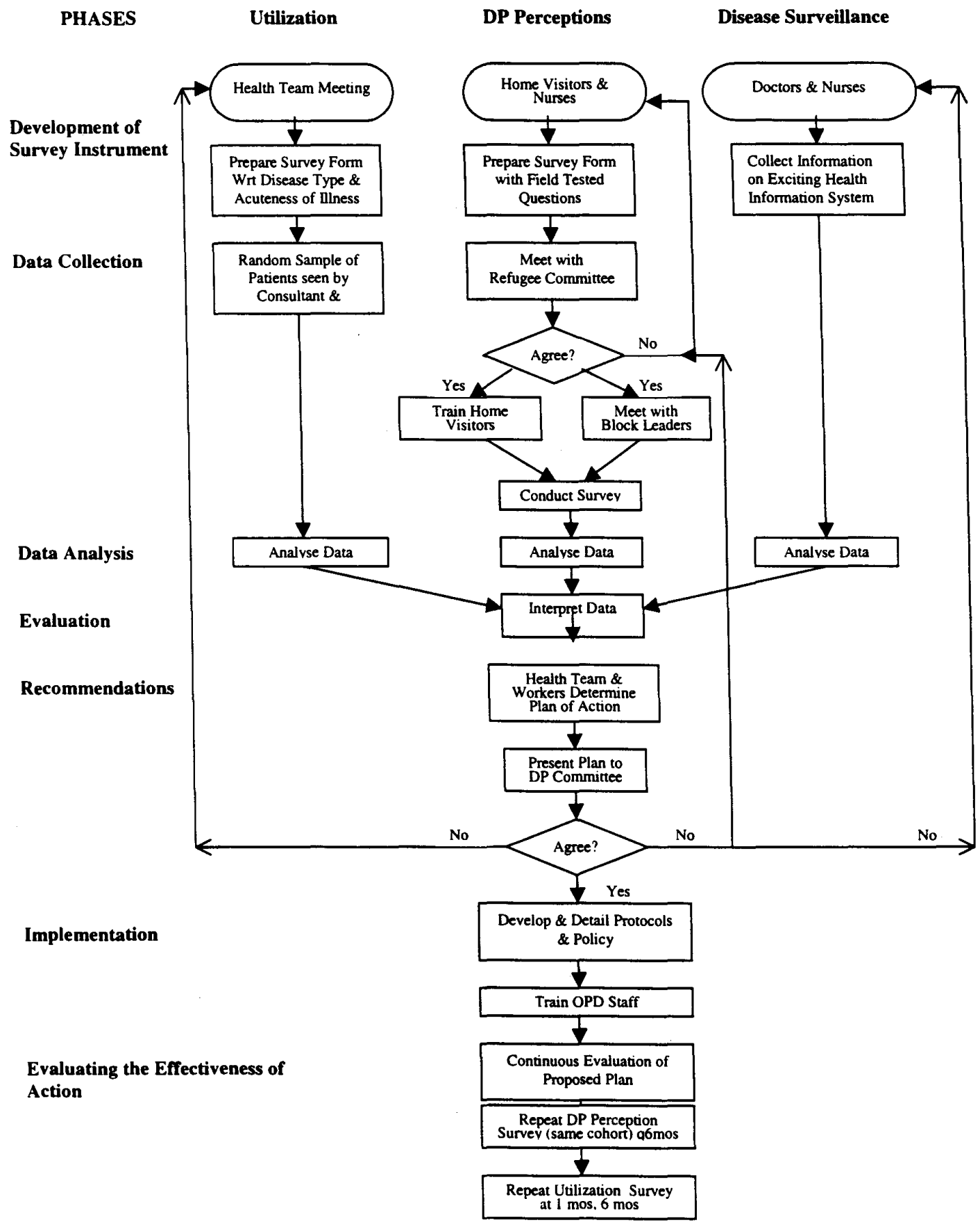

Refuge, Vol.18, No. 5 (January 2000)

(C) Paul B. Spiegel, Ellen Lynch and Narendra M. Patel, 2000. This open-access work is licensed under a Creative Commons AttributionNonCommercial 4.0 International License, which permits use, reproduction and distribution in any medium for non-commercial purposes, provided the original author(s) are credited and the original publication in Refuge: Canada's Journal on Refugees is cited. 


\section{Establishment of a General Framework to Improve Efriciency and Quality of OPDs in DP Camps}

After analyzing all of the above information, the team established a framework for meeting its stated objectives (Table 1). The group was not able to collect data as laid out in Chart 3, however, it created the framework based upon their numerous years of experience in DP health care.

\section{Short and Long Term Indicators}

Indicators with time limits must be agreed upon. Listed below are the types of short and long term indicators which would be appropriate in this case.

Short Term Indicators :

1. Decrease patient volume from a to $b$ patients/health care worker/day over 3 months.

2. Decrease medication usage by $x \%$ per day due to thesubsequent decreased patient volume, and the change in the manner of distribution of medications by the pharmacy over 3 months.
Long Term Indicators:

1. Decrease patient volume from $y$ to $\mathrm{z}$ patients/health care worker/day after 1 year.

2. Decrease medication usage by another $\mathrm{x} \%$ per day, after 1 year, due to changes in health care workers' prescribing practices and after analysis of disease surveillance and utilisation processes.

\section{Discussion}

Principles and tools of $\mathrm{CQI}$ have been applied to the general setting of a DP camp. The ultimate goal of the application of CQI is to improve the management and quality of care of an OPD, and thus ultimately decrease morbidity and mortality. This article should act as an introduction of CQI to administrators and HCWs working in DP camp settings. Furthermore, the CQI tools sited heremay act as templates to aid workers in the field.

The framework listed above (Table 1) is an outline which needs to be filled in according to the situation in the field. The CQI team re-engineered certain

Table 1:

\section{General Framework to Improve Efficiency and Quality of OPDs in DP Camps}

A. More efficient use of the Out Patient Department

1. Decrease overusage of repeat health care seekers:

i. Implement a new process to control access to OPD.

ii. Enforce rule that health card must be presented before delivery of care.

2. Change DP's and local person's expectations of the health care available: i. Decrease access to non-emergent care by local persons.

ii. Take a sample survey to assess DP's expectations of care to be provided and then then respond accordingly.

iii. Evaluate and prioritise medically beneficial and cost effective measures compared to DP's to expectations.

iv. Meet with DP leaders to create a plan and then disseminate it to residents and locals.

3. Improve quality of care:

i. Improve health information systems.

ii. Improve training of health care workers.

iii. Improve overall morale of staff in OPD.

B. Satisfy the health care needs of DPs given limited resources

1. Build support systems.

C. Prevent long term dependency in order to achieve repatriation

1. Regulate the care provided in OPD. processes of the composite camp in order to improve its efficiency and quality, which are listed below. In order to decrease overusage of repeat health care seekers by controlling access to the OPD, it was proposed that emergency cases would be seen 24 hours, 7 days per week while non-emergent case would be divided into new cases and chronic or follow up cases. New cases would be seen in the mornings with no limitation as to the number of cases seen by the HCWs. Chronic or follow up cases would be seen in the afternoons and would be limited to 20 patients/HCW. Furthermore, if a customer does nothave his issued health card, then he will not be seen until he returns to the OPD with it, unless it is an emergency. If the card is lost, then a new one will be issued but care will be delayed by one day for non urgent cases. To decrease patients being seen in the OPD for repeat psychosomatic illnesses, training of $\mathrm{HCWs}$ to provide small group counseling to specific groups, in order to treat these illnesses as well as to decrease clinic usage, should be implemented.

All critical customers should evaluate and prioritise medically beneficial and cost effective measures and compare them to the DP's expectations. For example, although some of the population may prefer injectable medications, the benefits of oral drugs may out weight their preference. For less severe cases, oral medications usually result in the same outcome with no secondary infections at the injection site or transmission of diseases from a contaminated needle, less discomfort to the patient, less medical waste to dispose of, and they are cheaper and do not require as much manpower.

Clinical diagnosis of malaria is neither sensitive nor specific (Meek et al. 1999) and thus whenever possible, a malaria smear should be taken before treatment. Random prescription of antimalarial drugs is expensive, promotes drug resistance, and has numerous side effects. Despitecustomer preference for antimalarial medications when fever and chills are present, the use of antipyretics should be weighed against antimalarial drugs for the non-vulner- 
ablegroups in a population at first presentation, when no lab is available to confirm the diagnosis. Patients must return to clinic in 24 hours for a followup and a system for finding patients who donot return mustbe in place before this system is initiated. Furthermore, certain expensive or desirable medications from the OPD, for instance quinine, are commonly sold in the local markets. One way to decrease this wastage of medication and improve compliance of treatment is to employ directly observed therapy for certain medications. The patient must return daily to the clinic or a home care visitor must observe the patient take the medicine at home. Once these decisions are made together with the community, DP leaders must create and then disseminate the plan to residents in the camp in order to seek its acceptance.

Quality of health care can be improved by improving the health information systems (Hakewill and Moren 1991). This process involves re-analysing the existing surveillance systems using a standard criteria for evaluation (CDC 1988, CDC 1992). Furthermore, case definitions and treatment protocols should be followed by HCWs. This requires patience and persistence on behalf of the clinical manager. Lastly, definition fornew (incident) versus repeat cases should be developed and implemented.

In order to improve the quality of care $\mathrm{HCWs}$ provide as well as improve the HCW's morale, the following processes were proposed: weekly lectures and preceptor sessions (to transfer skills and knowledge), weekly review of patient and medication registers, the provision of educational material (books, guidelines) and appropriate medical equipment, consistent feedback to the health team regarding their performance and programmatic changes initiated due to the data collection effort and reports provided. In addition routine meetings, assessments, positive reinforcement and team building should be implemented.

There is a fine line between attempting to offer health care to DPs in a comprehensive sense while being careful neither to offer too sophisticated care and thus create a disincentive towards repatriation, nor to create inequities compared to the services available to the local population (Allen and Morsink 1994, Allen 1996). In order to satisfy the health care needs as well as the expectations of the DPs, given the limited resources and proposed changes in OPD processes, support systems will need to be recognised and reinforced at all levels. Health information systems and customer exit surveys should be used to collect information regarding customer's usage and satisfaction of the clinic. This data should be analysed and acted upon accordingly.

Lastly, in order to prevent long term dependency by the DPs on the health care system, care provided in OPD should be regulated. Treatment should be restrict to the UN essential drug list. Guidelines for referrals to local or mission hospitals should be defined and strictly enforced. These restrictions should help reduce the reluctance of DPs to return to their place of origin when conditions are appropriate to do so, as occurred in Liberia (Schownegerdt 1998) and Cambodia.

Although this article is vertically focused on the functioning of OPDs in DP camps, it must be emphasised that at least part of the team assessing the OPD should also be used to assess other areas of health care provision in the camp (i.e. hospital, Mother-Child Health centre, feeding centers, and home visiting programs) to allow for a more comprehensive application of CQI towards the total health care provided to the DP population.

This article is limited in scope for several reasons. Firstly, the team conducted the analysis without the breadth of input from other key players in DP health care. Thus people involved in all stages of the actual OPD process, from health care workers to consumers to leaders of the camp to representatives of the host country, were not able to be involved in the analysis. Secondly, data from a specific camp were not utilised, which would have allowed for more specific recommendations. In lieu of these limitations, a composite camp composed of the most common processes and problems faced by OPDs in DP camps was developed.

\section{Conclusion}

Over the last two decades, many changes have occurred in DP health care, some due to the increase in experience and expertise developed in the field, and others due to changing political and economic climates. Continuous Quality Improvement can address most, if not all of these changes. By involving health care workers at all levels together with the patients and the community, in defining, analysing and realistically implementing cost-effective CQImeasures, improved confidence, communication, and a clearer understanding of the community's needs as well as the restrictions and limitations of the UN and NGO agencies will develop. Furthermore, HCW's morale should improve, increasing job satisfaction and improving the atmosphere within a difficult work environment. As with health care throughout the world, the quality and cost of medical care is no longer determined by health care providers and a few important stakeholders (i.e. NGOs or the UN) but increasingly by the consumers and funding agencies (i.e. DPs themselves and the donor agencies). NGOs and aid workers can no longer simply provide medical care with little or no accountability (Toole and Waldman 1993). Donor agencies and the DPs themselves are demanding that NGOs be held accountable for their performance and provide quantifiable measures and indicators of effectiveness. NGOs, the UN and HCWs cannot approach this new paradigm with outdated management structures(Bender 1995). The dramatically rising costs of aid for the international community due to the successively increasing number of ethnic conflicts over the past decade must be controlled. CQImay prove to be an effective method tolower health care costs in DP camps while improving the quality 
of care as well as patient, community and health care worker satisfaction.

This article showed how CQI tools may be applied in DP camps, as well as provided potential solutions towards improving the efficiency and quality of health care in an OPD. Thenextstep will be to implement this framework at the field level using all the appropriate levels of health care workers and critical customers. This stage will allow data to be collected and analysed using CQI tools and methods, allowing for specific solutions to be developed for each particular camp. Either a case-control study or a retrospective cohort study would be feasible and provide invaluable information on the actual process of implementation as well as possible benefits of applying CQI to a DP camp setting.

\section{Acknowledgements}

We are grateful to Drs. R. Morrow and G. Burnham of the department of International Health, Johns Hopkins School of Hygiene and Public Health for the invaluable help they each provided with the preparation of this manuscript.

\section{References}

Allen, T. 1996. In Search of Cool Ground: War, Flight \& Homecoming in northeast Africa, Africa World Press, Trenton, N.J.

Allen, T. and H. Morsink. 1994. When Refugees Go Home : African Experiences, Africa World Press, Trenton, N.J.

Bender, A. 1995. Organization of the Medical Practice and the Implications for Delivering Quality Care.,Quality Management in Health Care, 3, 47-53.

Bender, A. D. and C. J. Krasnick.1993. Applying TotalQuality Management to theMedical Practice.,Journal of Health Care Financing, 15, 22-6.

Brassard, M. and D. Ritter. 1994. The Memory Jogger II, GOAL/QPC Resource, Washington DC.

Brown, L.D., L. FrancoN. Rafeh and T. Hatzell (Ed.) .1992. Quality Assurance of Health Care in Developing Countries., Johns Hopkins University Press, Washington DC.

CDC. 1988. Guidelines for Evaluation of Surveillance Systems,Morbidity, Mortality Weekly Report, 37, 1-18.

CDC. 1992. Famine-affected, refugee, and displaced populations: recommendations for public health issues.,Morbidity, Mortality Weekly Report, 41, 1-76.
Deming, W. E. 1986. Out of the crisis, Massachusetts Institute of Technology Center for Advanced Engineering Study, Cambridge, Mass.

Desenclos, J. C. 1993. Clinical Guidelines: Diagnostic and Treatment Manual, Medecins Sans Frontieres, Paris.

Hakewill, P. A. and A. Moren. 1991. Monitoring and Evaluation of Relief Programmes, Trop Doct, 21, 24-8.

IDA. 1990. Basic Unit: Treatment Guidelines, International Dispensary Association, Amsterdam.

IFRC. 1996. World Disasters Report, International Federation of Red Cross, Oxford.

Médecins Sans Frontières. 1997. Refugee Health: An approach to emergency situations, MacMillan Education Limited, London.

Meek, S., M. Rowland and M. Connolly 1999. Outline of strategy for malaria control in complex emergencies, World Health Organization, Geneva, pp. 16.

\section{Summer Course on Refugee Issues}

\section{Toronto, June 11 - 18, 2000}

The Centrefor Refugreestudies: Summer Courseoffers postgrad wate training inrefugee issues for up to fíty practitioners inside and outside government who work on some aspect of refugee protection or assistance. The courre includes panel discussions, case studies a sinulation exercise and lectures from international experts. A York Untversity/Centre for Refugee Sturites Certificate is awarded upon successful completion of the seren day program Sessions offered in 2000 include:

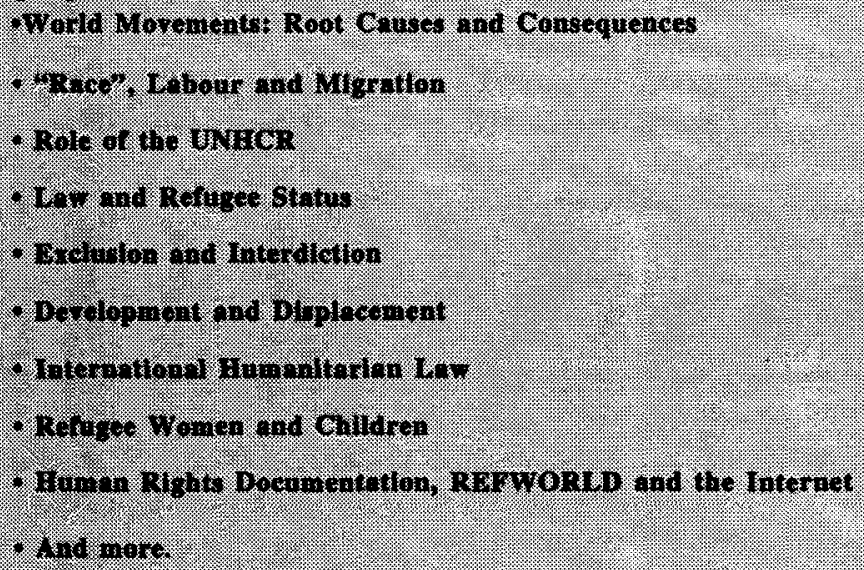

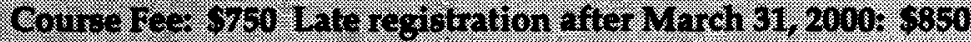

Fees are in Cenadian dollars and include materials. Foo $\mathrm{d}$ and $\mathrm{accomm}$. dation

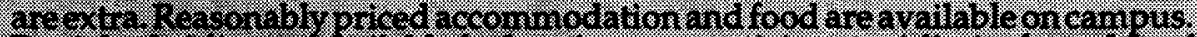
Partal subsidtes are avallable for low - ncome participants. Alimited number of

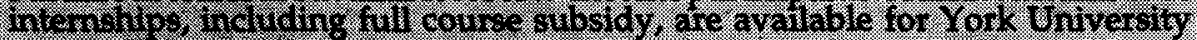
stadenk. Deadine Forgubsiay and internstip applications is February 1, 2000.

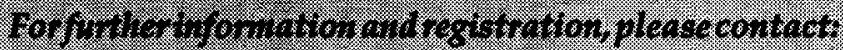

\section{Sharryn Aiken, Academic Director}

Centrefor Refuge-Studies, York Univeroity, Suite 329 , York Lanes,

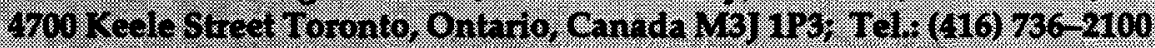

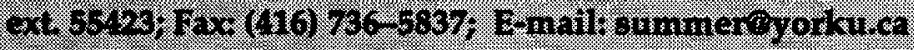

